\title{
Heat transfer and entropy generation in a microchannel with longitudinal vortex generators using nanofluids
}

Amin Ebrahimi ${ }^{1}$, Farhad Rikhtegar ${ }^{2}$, Amin Sabaghan $^{1}$, Ehsan Roohi ${ }^{1, *}$

1- High Performance Computing (HPC) Laboratory, Department of Mechanical Engineering, Faculty of Engineering, Ferdowsi University of Mashhad, Mashhad, P.O. Box 91775-1111, Iran.

2- Harvard-MIT Biomedical Engineering Center, Massachusetts Institute of Technology, 77 Massachusetts Avenue, Cambridge, Massachusetts 02139, United States.

* - Corresponding Author, Email: e.roohi@um.ac.ir, Phone: +989155153124, Address: High Performance Computing (HPC) Laboratory, Department of Mechanical Engineering, Faculty of Engineering, Ferdowsi University of Mashhad, Mashhad, P.O. Box 91775-1111, KhorasanRazavi, Iran. 


\section{Abstract}

Conjugated heat transfer and hydraulic performance for nanofluid flow in a rectangular microchannel heat sink with longitudinal vortex generators (LVGs) are numerically investigated using a finite-volume approach at different ranges of Reynolds numbers. Steadystate three-dimensional simulations are performed on a microchannel heated by a constant heat flux with a hydraulic diameter of $160 \mu \mathrm{m}$ and six pairs of LVGs using a single-phase model. Coolants are selected to be nanofluids containing low volume-fractions $(0.5 \%-3.0 \%)$ of $\mathrm{Al}_{2} \mathrm{O}_{3}$ or $\mathrm{CuO}$ nanoparticles with different particle sizes dispersed in pure water. The proposed model is validated and compared by already-published experimental, and single-phase and two-phase numerical data for various geometries and nanoparticle sizes. The comparison of results obtained from proposed single-phase model and two-phase model favours the former. The results demonstrate that heat transfer is enhanced by $2.29-30.63 \%$ and $9.44 \%-53.06 \%$ for water- $\mathrm{Al}_{2} \mathrm{O}_{3}$ and water- $\mathrm{CuO}$ nanofluids, respectively, in expense of increasing the pressure drop with respect to pure-water by $3.49 \%-16.85 \%$ and $6.5 \%-17.70 \%$, respectively. We have also observed that the overall efficiency is improved by $2.55 \%-29.05 \%$ and $9.78 \%-50.64 \%$ for water- $\mathrm{Al}_{2} \mathrm{O}_{3}$ and water-CuO nanofluids, respectively. The results are also analyzed in terms of entropy generation, leading to the important conclusion that using nanofluids as the working fluid could reduce the irreversibility level in the rectangular microchannel heat sinks with LVGs. No exterma (minimum) is found for total entropy generation for the ranges of parameters studied.

Keywords: Microchannel; Longitudinal vortex generators; Nanofluids; Fluid flow; Heat transfer; Entropy generation. 


\section{Introduction}

Over the past 35 years, the concept of microchannel heat sink, pioneered by Tuckerman and Pease [1], has received immense attention. Extensive usage of microchannels has played a basic role in electronics cooling applications, medical instruments, laser equipments, automotive industries, and aerospace technology among others. Flow and heat transfer in microchannels have been the focus of research by numerous investigators as reviewed by Morini [2], Agostani et al. [3] and Adham et al. [4]. Li et al. [5] numerically studied the laminar flow and heat transfer in microchannels for Reynolds numbers lower than 500 and reported that conventional Navier-Stokes and energy equations without slip boundary condition are valid for liquid flows in microchannels with a hydraulic diameter of tens of micrometers.

Different working fluids are employed in microchannels, wherein for heat sinks, liquid coolants are preferred over the gaseous coolants thanks to their higher heat transfer coefficients [6]. Still a poor thermal conductivity of common liquid coolants such as water, oil and organic chemicals such as ethylene glycol, compared to most solids, is a limiting factor to achieve high efficacy, lower weight and acceptable miniaturization of heat sinks. To circumvent the low thermal conductivity of conventional coolants, Masuda et al. [7] offered adding ultrafine solid particles suspansion to conventional working fluid in order to enhance the heat transfer performance. Later, this new type of coolants named as "nanofluid" by Choi [8] broadly accepted by the heat transfer society. Nanofluids are of great advantage thanks to their significantly-high thermal conductivity with good stability.

Mainstream of research so far has been on the investigation of thermo-physical properties of nanofluids with various types of nanoparticles and base-fluids [9-16]. It is well accepted that the thermal conductivity of all nanofluids is higher than that of the base fluids, and volumefraction, diameter, material and bulk temperature of nanoparticles play significant roles in determining the thermal conductivity of nanofluids. Part of available review papers are dedicated to the elaboration of the thermo-physical properties, flow characteristics and current stage of nanofluids [17-22]. In recent years, numerous experimental and numerical studies have been conducted mainly focusing on the efficacy of nanofluid usage in heat transfer augmentation for microchannels due to the very promising performance of nanofluids on heat transfer enhancement. Kalteh et al. [23] numerically investigated laminar water-Cu nanofluid flow and forced convective heat transfer inside a microchannel with constant wall temperature using a two-phase model. Their results indicate that the heat transfer performance rises, increasing particle volume fraction and Reynolds number and decreasing nanoparticle size at the expense of a higher pressure drop. In another work Kalteh et al. [24] studied the hydrodynamic and thermal behavior of water-alumina nanofluid flowing through a wide microchannel heat sink both numerically and experimentally and negligible temperature and velocity differences between the phases are reported. The heat transfer performance of a circular microchannel heat sink is studied numerically for three different nanofluids by Sohel et al. [25]. Superior heat transfer performance is observed in electronics cooling applications using water- $\mathrm{Cu}$ nanofluid compared to water-alumina and water- $\mathrm{TiO}_{2}$ nanofluids. The increased pressure drop, due to the higher viscosity of nanofluids, is ignored by Sohel et. al. Mital [26] proposed a model using semi-empirical correlations to evaluate nanofluid 
thermophysical properties. His statistical analysis of the proposed model indicates that the thermal performance of microchennal heat sinks, mostly depends on nanoparticle volumefraction, followed by the nanoparticle diameter, wherein smaller particles result in higher heat transfer improvement without any significant increase in pressure drop. Furthermore, comparatively weak dependance is reported between the Reynolds number variation and thermal performance. Mital also proposed an optimum volume-fraction for any Reynolds number which maximizes the heat transfer enhancement with smallest possible particle size. Rimbault et al. [27] performed an experimental study on heat transfer and water-CuO nanofluid flow characteristics in an isothermal and heated rectangular microchannel under both laminar and turbulent flow regimes. They reported better heat transfer performance with a higher pressure penalty with respect to water flow. They also reported a comparable critical Reynolds number of 1000 both for water and nanofluids. To improve the heat transfer performance of metallic nanofluids, Nimmagadda and Venkatasubbaiah [28] numerically studied the laminar forced convection of hybrid nanofluids in a wide microchannel. They compared different kinds of nanofluids and observed a heat transfer enhancement of $143 \%$ using hybrid nanofluids with respect to pure water. The boundary-layer flow as a fundamental problem is recently revisited to explore the effects of nanofluids use on flow patterns,thermal fields and the entropy generation behavior [29, 30]. Khamis et al. [31] numerically investigated the variable viscosity of nanofluid flow and its subsequent effect on heat transfer in a porous pipe, reporting that both fluid temperature and velocity increase reducing the viscosity.

Using novel techniques of heat transfer enhancement, classified into active and passive methods [32, 33], design of smaller, lighter, more efficient and portable heat transfer equipments will be possible. In 1969, Johnson and Joubert [34] used Vortex Generators (VGs), a case in point in passive approach, to improve the heat transfer for the first time. The flow separation from the side edges of VGs generates longitudinal, transverse and horseshoe vortices which form secondary flow, intensify fluid mixing and distort the thermal boundary layer causing higher heat transfer rate [35-37]. At small attack angles of VGs the mainly longitudinal vortices are dominant which potentially could lead to greater heat transfer augmentation with less required pumping power compared to transverse vortices generated at high attack angles of VGs [38]. VGs can be employed in different forms, including fins, wings, ribs, winglets and protrusions [39]. There has been recently much higher interest in the applications of VGs in microchannels. Liu et al. [40] experimentally investigated the heat transfer and pressure loss in microchannels with longitudinal vortex generators (LVGs). Decreased critical Reynolds number to 600-730 is reported adding LVGs compared to smooth microchannel accompanied by better heat transfer performance and higher flow loss. Lan et al. [41] numerically studied the effects of dimple/protrusion arrangement, Reynolds number and streamwise pitch on heat transfer performance in a rectangular microchannel with water as a coolant under laminar flow condition. They found better thermal performance with low pressure loss using the dimple/protrusion technique in mcirochannels. More recently, Mirzaee et al. [42] used elastic VGs to improve heat transfer in microchannels. They observed $15-35 \%$ increase in the mean Nusselt number and a 10-70\% increase in the friction factor in the laminar flow regime. They also state that using rigid VGs results in lower Colburn/friction factor ratio compared to elastic VGs. Chen et al. [43] by extending the experimental study of Liu et al. [40] examined various 
VGs in microchannels with different hydraulic diameters. To enhance the heat transfer performance of microchannel heat sinks, $\mathrm{Li}$ et al. [44] used water-alumina nanofluids in the microchannel with dimple/protrusion. To better understand heat transfer characteristics and flow behavior in microchannels with LVGs, Ebrahimi et al. [45] numerically investigated the laminar water flow in microchannels with various configurations. They evaluated the overall efficiency of microchannels with LVGs considering both heat transfer and friction factor characteristics and found generally higher efficiency except for one particular case at Reynolds number of 100 .

Nanofluids, as very promising coolants, may improve the heat transfer efficiency of microchannels with LVGs. In order to explore in more depth the effects of using nanofluids on flow and conjugated heat transfer characteristics, different nanofluids with various particle diameters, material and concentrations are considered in the present study. Therein, threedimensional simulations are carried out using validated and efficient thermal conductivity and viscosity models which include the effects of nanoparticle size, bulk temperature as well as the Brownian motion of particles. The obtained results are compared with water as the plain working fluid for different Reynolds numbers under laminar flow condition. Additionally, the thermodynamics performance of the device is studied using entropy generation analysis. According to the reviewed papers and to the best of the authors' knowledge the combination of LVGs and nanofluids in microchannels is not addressed yet and the up-coming results may introduce new perspectives towards novel methods of heat transfer augmentation in microchannels.

\section{Physical model and numerical method}

\subsection{Geometrical configuration and computational domain}

Three-dimensional simulations are performed on a microchannel with LVGs to scrutinize the effects of using nanofluids on conjugated heat transfer and flow behavior. The schematic diagram of the physical model and relevant geometrical parameters are shown in Fig.1, and the details of geometric parameters are presented in Table 1. A Cartesian coordinate system is employed for describing the fluid flow, in which $z$ axis stands for stream-wise direction. According to Fig.1, the computational domain consists of three zones. The inlet block, with the length of $\mathrm{L}_{\text {in }}$ including adiabatic walls, represents the flow developing zone. The outlet block, with the length of Lout including adiabatic walls, is created to avoid any possible backflow that may influence the accuracy of the ultimate results. Finally, in the middle, six equally spaced pairs of LVGs are located at the heated region of the michrochannel (with the length of $\mathrm{L}_{\mathrm{ht}}=100 \mathrm{H}$ ). Different standard etching processes could be employed to manufacture the designed heat sink $[43,46]$. In order to decrease the computational costs, only the hatched region in Fig. 1 (a) is considered for numerical simulations because of symmetric arrangements of microchannel and LVGs.

\subsection{Mathematical models, governing equations and boundary conditions}

The microchannel and LVGs in the present study are assumed to be made of silicon, a conductive material, and the effects of surface roughness are neglected [40, 43, 45]. Pure-water and various nanofluids with low volume-fraction and various sizes of $\mathrm{Al}_{2} \mathrm{O}_{3}$ or $\mathrm{CuO}$ 
nanoparticles dispersed in pure-water are selected as coolants. A single-phase model is adopted here to effectively predict the heat transfer performance of nanofluids. The single-phase model is deemed to be valid because of both low nanoparticles concentration and diameters smaller than $100 \mathrm{~nm}[47,48]$. The thermo-physical properties of coolants are functions of temperature, size and volume fraction of nanoparticles and assumed to behave Newtonian. In addition, the coolant flow is assumed to be laminar due to the low fluid velocity and small pitch of the LVGs. Generation of longitudinal vortices is also reported to be a quasi-steady phenomenon [49]. Furthermore, in this study the effects of compressibility, body forces and radiation are neglected. Based on the these assumptions, the continuity, momentum, and energy equations for a steady-state flow can be written, respectively, as [45]:

$$
\begin{aligned}
& \nabla \cdot \vec{V}=0 \\
& \rho \vec{V} \cdot \vec{W}=-\nabla p+\nabla(\mu \vec{W}) \\
& \rho c_{p}(\vec{V} \cdot \nabla T)=\nabla(k \nabla T)+\Phi
\end{aligned}
$$

wherein $\vec{V}$ is velocity vector, $p$ is static pressure, $T$ is temperature, $\rho$ is density, $k$ is thermal conductivity, $c_{p}$ is specific heat capacity, $\mu$ is dynamic viscosity and ultimately $\Phi$ corresponds to the dissipation function expressed as:

$$
\begin{aligned}
\Phi= & \left\{2\left[\left(\mu \frac{\partial u}{\partial x}\right)^{2}+\left(\mu \frac{\partial v}{\partial y}\right)^{2}+\left(\mu \frac{\partial w}{\partial z}\right)^{2}\right]+\right. \\
& \left.\left(\mu \frac{\partial u}{\partial y}+\mu \frac{\partial v}{\partial x}\right)^{2}+\left(\mu \frac{\partial v}{\partial z}+\mu \frac{\partial w}{\partial y}\right)^{2}+\left(\mu \frac{\partial w}{\partial x}+\mu \frac{\partial u}{\partial z}\right)^{2}\right\}
\end{aligned}
$$

additionally, for the solid zone, the energy equation is given as:

$$
\nabla^{2} T_{s}=0
$$

According to Fig.1 (b), a constant and uniform velocity is imposed at the channel inlet (face: 1-2-3-4) in the $z$ direction and its temperature is fixed at $298 \mathrm{~K}$. The gradients of dependent variables, i.e. $u, v, w$ and $T$, are set to zero at the outlet boundary (13-14-15-16). A uniform constant heat flux of $q_{w}^{\prime \prime}=20 \mathrm{~W} / \mathrm{cm}^{2}$ with no-slip boundary conditions are applied to the top wall of the heated zone (5-8-14-9). A symmetric boundary condition is defined on the rightside of the computational domain (4-16-15-3). Eventually, non-slip and adiabatic boundary conditions are applied to all the remaining faces of the microchannel. The boundary condition at the solid-liquid interfaces, i.e. LVGs surfaces, is introduced as follows:

$$
k \frac{\partial T}{\partial \vec{n}}=k_{s} \frac{\partial T_{s}}{\partial \vec{n}}
$$

Where, $\vec{n}$ is a normal vector on the solid-liquid interface drawn outward the boundary. The subscript $s$ represents solid characteristics. 
The thermo-physical properties of pure-water, silicon, $\mathrm{Al}_{2} \mathrm{O}_{3}$ and $\mathrm{CuO}$ nanoparticles are presented in Table 2. The effective thermo-physical properties of nanofluids are calculated using available correlations and are presented below.

The density of nanofluid is obtained based on the mixture model as follows [53]:

$\rho_{n f}=(1-\alpha) \rho_{b f}+\alpha \rho_{n p}$

wherein the subscript $n f, b f$ and $n p$ stand for nanofluid, base-fluid and nanoparticle, respectively, and $\alpha$ is the volume-fraction of nanoparticles.

The specific heat of nanofluid is determined by assuming thermal equilibrium between the primary fluid and nanoparticles [54].

$c_{p, n f}=\frac{(1-\alpha)\left(\rho c_{p}\right)_{b f}+\alpha\left(\rho c_{p}\right)_{n p}}{(1-\alpha) \rho_{b f}+\alpha \rho_{n p}}$

The effective thermal conductivity and dynamic viscosity of nanofluids are acquired by considering the effect of Brownian-motion of nanoparticles using a validated model based on experimental datasets [55, 56]. This model is valid for volume-fractions up to $4 \%$ and a temperature range between $300 \mathrm{~K}$ and $325 \mathrm{~K}$. The relation of the effective thermal conductivity of nanofluid reads as:

$k_{\text {eff }, n f}=k_{\text {static }}+k_{\text {Brownian }}$

where $k_{\text {static }}$ and $k_{\text {Brownian }}$ could be obtained from Hamilton-Crosser model [57] and kinetic theory, respectively.

$$
\begin{aligned}
& k_{\text {static }}=k_{b f}\left[\frac{\left(k_{n p, e f f}+2 k_{b f}\right)-2 \alpha\left(k_{b f}-k_{n p, e f f}\right)}{\left(k_{n p, e f f}+2 k_{b f}\right)-\alpha\left(k_{b f}-k_{n p, e f f}\right)}\right] \\
& k_{\text {Brownian }}=5 \times 10^{4} \alpha c_{p, b f} \rho_{b f} \sqrt{\frac{K_{b} T}{d_{n p} \rho_{n p}}} g\left(T, \alpha, d_{n p}\right)
\end{aligned}
$$

in which $K_{b}$ is Boltzmann constant, $d_{n p}$ is nanoparticle diameter. In order to take into account the effect of the interfacial thermal resistance, $k_{n p, e f f}$ can be determined as:

$$
k_{n p, e f f}=\frac{d_{n p}}{R_{b}+\left(d_{n p} / k_{n p}\right)}
$$

where $R_{b}$ is Kapitza resistance and is equal to $4 \times 10^{-8} \mathrm{~km}^{2} / W$.

The effective viscosity of nanofluids can be predicted as the sum of static and Brownian viscosity defined by the following equations.

$$
\mu_{e f f, n f}=\mu_{\text {static }}+\mu_{\text {Brownian }}
$$




$$
\mu_{\text {static }}=\frac{\mu_{b f}}{(1-\alpha)^{2.5}}
$$

$$
\mu_{\text {Brownian }}=5 \times 10^{4} \alpha \rho_{b f} \sqrt{\frac{K_{b} T}{d_{n p} \rho_{n p}}} g\left(T, \alpha, d_{n p}\right)
$$

The function $g$, a semi-empirical relation, is defined in bellow format for water-CuO and water$\mathrm{Al}_{2} \mathrm{O}_{3}$ nanofluids by fitting experimental data sets with regression values of $98 \%$ and $96 \%$, respectively [58].

$$
\begin{aligned}
g= & {\left[a+b \ln \left(d_{n p}\right)+c \ln (\alpha)+d \ln (\alpha) \ln \left(d_{n p}\right)+e \ln \left(d_{n p}\right)^{2}\right] \ln (T) } \\
& +\left[m+h \ln \left(d_{n p}\right)+i \ln (\alpha)+j \ln (\alpha) \ln \left(d_{n p}\right)+k \ln \left(d_{n p}\right)^{2}\right]
\end{aligned}
$$

The proposed models to obtain the nanofluid thermo-physical properties are previously utilized and validated against experimental data sets by different researchers [47, 48].

\subsection{Numerical solution and data reduction}

In the present study, a commercial package for computational fluid dynamics based on the finite volume method (Fluent) is used. A structured non-uniform grid is used with considerably-refined cells near the LVGs and channel walls to discretize the computational domain. The SIMPLEC algorithm is utilized to model pressure-velocity coupling. Herein residues of $1.0 \times 10^{-6}, 1.0 \times 10^{-6}$ and $1.0 \times 10^{-8}$ are defined as convergence criteria for the momentum, continuity and energy equations, respectively. The following parameters are exploited to construct a framework of ultimate result presentation. The Reynolds number $(R e)$ and the hydraulic diameter $\left(D_{h}\right)$ of the microchannel are defined as follows:

$$
\begin{aligned}
& R e=\frac{\rho_{i n} V_{i n} D_{h}}{\mu_{i n}} \\
& D_{h}=\frac{2 W H}{W+H}
\end{aligned}
$$

where $V$ is fluid velocity and subscript in stands for the inlet condition. The heat transfer coefficient $(h)$ and the Nusselt number $(\mathrm{Nu})$ can be calculated using following relations [24, 48]:

$$
\begin{aligned}
& h=\frac{q^{\prime \prime}}{T_{\text {wall }, \text { avg }}-\left(T_{\text {in }}+T_{\text {out }}\right) / 2} \\
& N u=\frac{h D_{h}}{k_{b f, \text { avg }}}
\end{aligned}
$$

wherein $q^{\prime \prime}$ is the heat flux, $T_{\text {wall,avg }}$ represents the mean wall temperature and subscript out stands for the outlet condition. To calculate $\mathrm{Nu}$, the thermal conductivity $(k)$ is measured at the arithmetic mean temperature of the inlet and the outlet. The Fanning friction factor $(f)$ is defined by subsequent relation: 
$f=\frac{2 \Delta p}{\rho V_{i n}^{2}} \frac{D_{h}}{L}$

wherein

$$
\Delta p=\left(\bar{p}_{\text {out }}-\bar{p}_{\text {in }}\right)
$$

and $L$ is the stream-wise length of the microchannel and $\bar{p}$ is area-weighted static pressure. To attain the overall performance of the system the following relation is used:

$\eta=\left(\frac{N u}{N u_{b f}}\right)\left(\frac{f_{b f}}{f}\right)^{1 / 3}$

where $N u_{b f}$ and $f_{b f}$ represent mean Nusselt number and Fanning friction factor of the microchannel with pure-water as coolant. This performance parameter has been frequently employed to introduce the thermal performance of micro-devices dual benefiting from considering both the friction loss augmentation and the heat transfer enhancement $[44,45,59$, 60].

To evaluate the thermodynamic gain of using nanofluids as a coolant in the considered microchannel with LVGs the total volumetric entropy generation rate $\left(\dot{S}_{g e n}^{\prime \prime \prime}\right)$, which contains the entropy generations due to fluid frictional $\left(\dot{S}_{g, F F}^{\prime \prime \prime}\right)$ and heat transfer $\left(\dot{S}_{g, H T}^{\prime \prime \prime}\right)$ effects, is defined as $[61,62]$ :

$\dot{S}_{g e n}^{\prime \prime \prime}=\dot{S}_{g, F F}^{\prime \prime \prime}+\dot{S}_{g, H T}^{\prime \prime \prime}$

$\dot{S}_{g, F F}^{\prime \prime \prime}=\frac{\mu}{T}\left(\frac{\partial u_{i}}{\partial x_{j}}+\frac{\partial u_{j}}{\partial x_{i}}\right) \frac{\partial u_{i}}{\partial x_{j}}$

$\dot{S}_{g, H T}^{\prime \prime \prime}=\frac{k}{T^{2}}\left[\left(\frac{\partial T}{\partial x}\right)^{2}+\left(\frac{\partial T}{\partial y}\right)^{2}+\left(\frac{\partial T}{\partial z}\right)^{2}\right]$

The dimensionless total entropy generation $\left(S_{n}\right)$, frictional entropy generation $\left(S_{n, F F}\right)$ and entropy generation induced by heat transfer $\left(S_{n, H T}\right)$ are defined here in order to estimate theirreversibility. Furthermore, to assess the contribution of heat transfer and fluid friction in total irreversibility, a non-dimensional parameter, Bejan number $(\mathrm{Be})$, can be expressed as:

$$
\begin{aligned}
& S_{n}=\dot{S}_{g e n}^{\prime \prime \prime} \frac{k T_{i n}^{2}}{q_{w}^{\prime \prime 2}} \\
& S_{n, F F}=\dot{S}_{g, F F}^{\prime \prime \prime} \frac{k T_{i n}^{2}}{q_{w}^{\prime 2}}
\end{aligned}
$$


$S_{n, H T}=\dot{S}_{g, H T}^{\prime \prime \prime} \frac{k T_{i n}^{2}}{q_{w}^{\prime \prime 2}}$

$B e=\frac{\dot{S}_{g, H T}^{\prime \prime \prime}}{\dot{S}_{g e n}^{\prime \prime \prime}}$

\subsection{Grid independency and model validation}

To ensure the simulation accuracy keeping computational costs at bay, four grids with different sizes are considered for grid independence test. The test is performed under the highest Reynolds number (i.e. $R e=250$ ) with pure-water as coolant. Table 3 illustrates the obtained results for different grid sizes and their relative differences. Since the relative differences of $\mathrm{Nu}$ and $f$ are lower than $0.24 \%$ and $0.02 \%$, respectively, increasing the grid size from 1.0 million to 1.5 million, the former grid with fewer computational cells is selected for the simulations.

Experimental [63] and numerical [64] data for water as well as $1.0 \%$ and $2.0 \% \mathrm{Al}_{2} \mathrm{O}_{3}$-water nanofluid flowing through a rectangular microchannel with a hydraulic diameter of $341 \mu \mathrm{m}$, are used to validate the employed numerical setup. In the reference investigation, the coolant enters the microchannel with a temperature of $303.15 \mathrm{~K}$ and a constant heat flux of $22.32 \mathrm{~W} / \mathrm{cm}^{2}$ is imposed on the bottom wall, while other walls treated as adiabatic. According to the symmetric configuration of the channel, only half of the channel is modelled. Fig. 2 (a) and Fig. 2 (b) compare the present numerical results with the experimental [63] and single-phase and two-phase numerical [64] estimates for heat transfer coeficient and Fanning friction factor, respectively. It is worth mentioning that Ghale et al. [64] have used constant thermophysical properties in their models and have ignored the effects of Brownian-motion of nanoparticles in their single-phase model. Further validation of present numerical simulations has been done against experimental data of Lai et al. [65] for the study of thermal performance of water and $\mathrm{Al}_{2} \mathrm{O}_{3}$-water nanofluids in a laminar thermally developing tube flow. Fig. 2 (c) compares numerical predictions and experimental results of local heat transfer coefficients in the thermal developing region of a straight tube made out of AISI 316 stainless steel with $1.02 \mathrm{~mm}$ diameter and $50 \mathrm{~cm}$ long. The tube is under constant wall heat flux condition and the coolants flow through it at a volume flow rate of $1 \mathrm{ml} / \mathrm{min}$. It is clearly seen that the present numerical results could successfully predict the experimental data with an acceptable range of deviation. These small deviations may be attributed to several factors among which we can mention the simplifications of the physical model, the precision of the thermo-physical models, nonuniformity of nanoparticle sizes and ultimately the experimental measurements limits, nanoparticle distribution and the nanoparticle production method. Comparing results obtained from current single-phase model with two-phase model favours the proposed model. Additionally, two-phase models are more sophisticated and impose considerably higher computational costs; therefore, it is preferable to employ single-phase model, instead. It is also reported in recently published literature that a suitable viscosity-conductivity combination for the single-phase model could result more accurate predictions compared to two-phase models [66-68]. 


\section{Results and discussions}

Conjugated heat transfer and hydraulic effects of different nanofluids on the performance of microchannel heat sink with LVGs, under steady-state laminar flow regime and heated by constant heat flux, are analyzed based on proposed models.

Fig. 3 illustrates the effects of nanoparticle material and volume-fraction on the $N u$ and $f$ for various $R e$ and particle diameter of $29 \mathrm{~nm}$. A direct relation between $N u$ and $R e$ is observed for all coolants studied here. We argue that enlarging the recirculation zones behind the LVGs, enhancing the fluid mixing and decreasing the thermal boundary layer thickness as a result of $R e$ rise may be the root causes of better heat transfer performance at higher $\operatorname{Re}[45]$. We have observed that using nanofluids with low-volume-fractions of nanoparticles $(0.5 \%-3 \%)$ leads to a significant increase in $\mathrm{Nu}$ at the expense of slightly higher pressure loss. Furthermore, higher heat absorption is noticed for nanofluids which is mainly caused by higher thermal conductivity of the mixture of base-fluid and nanoparticles, larger heat transfer surface area and higher collision rate of nanoparticles giving rise to the Brownian motion of nanoparticles. The effects of motion mechanisms of other nanoparticle on thermal conductivity of nanofluids, e.g. thermo-phoresis and osmo-phoresis, could be neglected compared to the effects of Brownian motion [69]. The above-mentioned increase in thermal conductivity eventuates in intensifying the energy transfer owing to diffusion in the coolant and causing higher bulk temperatures. More heat transfer enhancement at higher volume-fractions is attributed to the higher momentum of nanoparticles compared to base-fluid molecules resulting in more efficient thermal-energy transfer [48] and higher thermal conductivity of the coolant. It is found that $\mathrm{Nu}$ is more sensitive to the volume-fraction of nanoparticles at lower $R e$. The rational behind this observation is that at lower $R e$ the bulk flow temperature is higher causing higher thermal conductivity and lower fluid viscosity. On the other hand, increasing the volume-fraction of nanoparticles brings about higher thermal conductivity and viscosity producing smoother temperature gradients. These effects increase the conduction heat transfer contribution in the total heat transfer budget, which is more dominant at lower $R e$. It is also figured out that water$\mathrm{CuO}$ shows higher heat transfer augmentation compared to water- $\mathrm{Al}_{2} \mathrm{O}_{3}$ at similar volumefraction and $R e$; because of higher thermal conductivity of $\mathrm{CuO}$ nanoparticles compared to $\mathrm{Al}_{2} \mathrm{O}_{3}$ nanoparticles as well as the effects of nanoparticles alignment, structure and interactions.

Based on Fig. 3, despite having better heat transfer performance, it is found that the Fanning friction factor may increase with an increase in nanoparticle volume-fraction, due to the higher viscosity of nanofluids with respect to the base-fluid. Actually, stronger hydrodynamic interactions between nanoparticles make nanofluids more viscous at a higher volume-fractions. At similar $R e$ and volume-fraction, water- $\mathrm{CuO}$ nanofluids bring about more pressure drop compared to water- $\mathrm{Al}_{2} \mathrm{O}_{3}$ nanofluids, wherein the Fanning friction factor is more affected by the nanoparticle volume-fraction at lower $R e$. For the nanofluids studied here, increasing $R e$ introduces lower $\mu_{\text {Brownian }}$ and higher $\mu_{\text {static }}$. The relative increase of the nanofluid viscosity with respect to the pure-water is greater at lower $R e$ causing even more pressure drop for a constant volume-fraction. In addition, more pumping power is required to drive nanofluids into microchannels at a higher volume-fraction of nanoparticles. Interestingly, when compared to the base-fluid, nanofluids including $0.5 \%$ volume-fraction of $\mathrm{Al}_{2} \mathrm{O}_{3}$ show slightly lower 
Fanning friction factor over the entire ranges of the $R e$. Same is observed for nanofluids including $0.5 \%$ volume-fraction of $\mathrm{CuO}$ for $R e>75$. This is due to the fact that for low concentration of nanoparticle the effective viscosity slightly increases introducing higher pressure drop; however, higher inlet velocity is required to achieve similar $R e$ compared to the nanofluids with more densely-concentrated nanoparticle because of the lower increase in effective density which eventually decreases the value of Fanning friction factor.

$\mathrm{Al}_{2} \mathrm{O}_{3}$ and $\mathrm{CuO}$ nanoparticles with variety of sizes are considered in the present study. The effects of nanoparticle size on heat transfer and hydraulic performance are shown in Fig. 4 for different $R e$. In case of water- $\mathrm{Al}_{2} \mathrm{O}_{3}$ nanofluid, decreasing the particle diameter leads to higher heat transfer rate as a result of higher heat transfer surface area, aggregation of nanoparticles and more intensified Brownian motion causing higher thermal conductivity of coolant. Smaller particles ensue more particles at a fixed volume-fraction, which increases the total surface area of nanoparticles, and therefore forms more area of nanolayers around particles eventually rising the thermal conductivity of nanofluid. Furthermore, more intensified Brownian motion amplifies the micro-mixing processes and consequently augments the thermal conductivity of the nanofluid. In contrast to water- $\mathrm{Al}_{2} \mathrm{O}_{3}$, thermal performance of water-CuO improves by increasing the nanoparticle size. This heat transfer enhancement is related to the higher thermal conductivity of water- $\mathrm{CuO}$ nanofluid with larger nanoparticles. Methods of producing nanoparticles, the stability of the suspension and test conditions have been introduced as principal reasons for the mentioned deviation from water- $\mathrm{Al}_{2} \mathrm{O}_{3}$ [47]. Thermal conductivity enhancement with increasing the $\mathrm{CuO}$ particle diameter may also be attributed to effects of aggregation and percolation [70-72]. Decreasing the particle size at a fixed volume-fraction results in close-alignment of particles attracting one another with greater strength due to the van der Waals forces [73] making larger aggregates. This so called larger aggregates not only increase the percolation effects, but also cause nanoparticle agglomeration and weaken the Brownian motion most probably leading to lower thermal conductivity. The different behavior of water- $\mathrm{Al}_{2} \mathrm{O}_{3}$ and water-CuO with changing the particle size may arise from dissimilar aggregation treatment owing to different nanoparticle charge, surfactant and zeta potential [74].

Fig. 4 also depicts that decreasing the size of $\mathrm{Al}_{2} \mathrm{O}_{3}$ nanoparticles has an adverse effect on hydrodynamic performance by increasing the device pressure loss. Including more nanoparticles by reducing the particle size at a constant volume-fraction initiates stronger interaction between particles rising the viscosity of the nanofluid; thereby, higher pressure drop is observed decreasing the size of $\mathrm{Al}_{2} \mathrm{O}_{3}$ nanoparticles. For water-CuO it is not the case as reducing the particle sizes results in lower pressure drop. The reason for this counter intuitive trend could be addressed by different aggregation behavior of $\mathrm{Al}_{2} \mathrm{O}_{3}$ and $\mathrm{CuO}$ nanoparticles. For the same particle size, $\mathrm{CuO}$ nanoparticles tend to make larger aggregates compared to $\mathrm{Al}_{2} \mathrm{O}_{3}$ nanoparticles. The agglomeration of smaller $\mathrm{CuO}$ nanoparticles presents lower viscosity, hence the need for lesser pumping power.

The effects of different nanofluids with various nanoparticle material, concentration and diameter on flow and thermal fields at $R e=150$ are shown in Fig. 5. In this figure $k_{m, n f}$ and $\mu_{m, n f}$ represent the mean nanofluid thermal conductivity and viscosity, respectively. It is clearly seen that for comparable nanoparticle diameter and concentration the water- $\mathrm{CuO}$ nanofluid brings 
higher thermal conductivity and viscosity compared to water- $\mathrm{Al}_{2} \mathrm{O}_{3}$ nanofluids, wherein this higher thermal conductivity results in higher bulk coolant temperature. We believe water-CuO nanofluids are more efficient than water- $\mathrm{Al}_{2} \mathrm{O}_{3}$ nanofluids for heat transfer enhancement applications. Higher nanoparticle volume-fraction also leads to higher thermal conductivity and viscosity for both water- $\mathrm{Al}_{2} \mathrm{O}_{3}$ and water-CuO nanofluids. Employing larger $\mathrm{Al}_{2} \mathrm{O}_{3}$ and smaller $\mathrm{CuO}$ nanoparticles in nanofluids will bring about better heat transfer performance due to higher thermal conductivity and smoother temperature gradients.

The results demonstrate a significant increase of $19.14 \%-53.06 \%$ and $9.79 \%-30.63 \%$ in Nusselt number vis-à-vis pure water for water-CuO and water- $\mathrm{Al}_{2} \mathrm{O}_{3}$, respectively, with $d_{p}=29 \mathrm{~nm}$. However, This enhanced heat transfer performance costs more demanded pumping power. Fig. 6 illustrates the overall efficiency of the microchannel with $\mathrm{LVGs}$ for water-CuO and water$\mathrm{Al}_{2} \mathrm{O}_{3}$ nanofluids. Using nanofluids as a coolant in microchannels with LVGs leads to a noticeable enhancement in overall efficiency which will be boosted even more by increasing the nanoparticle concentration (Fig. 6a). This figure also shows that for all the volumefractions, overall efficiency decreases with increasing Re. According to Fig. 6b, higher overall efficiency is observed for the smaller $\mathrm{Al}_{2} \mathrm{O}_{3}$ and larger $\mathrm{CuO}$ nanoparticles. Thus far among all the cases studied in the present investigation, water- $\mathrm{CuO}$ nanofluid with $d_{p}=29 \mathrm{~nm}$ and highest nanoparticle concentration shows the best overall efficiency. Furthermore, adding $1 \%$ volumefraction of $\mathrm{CuO}$ nanoparticles in the base-fluid results in a comparable overall efficiency with $3 \%$ concentration of $\mathrm{Al}_{2} \mathrm{O}_{3}$ nanoparticles, both with $d_{p}=29 \mathrm{~nm}$.

The generation of entropy wastes the available energy and causes a decline in efficiency by making losses in the profitable power. The dimensionless entropy generation as a function of Reynolds number for different nanoparticle materials, sizes and concentrations is shown in Fig. 7. Lower values of $S_{n}$ indicate better thermodynamic performance due to lower irreversibility in the system. It is observed that using nanofluids causes a reduction in entropy generation or irreversibility in microchannels with LVGs in comparison with water as a coolant. From Fig. 7, for a specified Reynolds number the highest and lowest values of $S_{n}$ belong to water- $\mathrm{Al}_{2} \mathrm{O}_{3}$ with $d_{p}=47 \mathrm{~nm}$ and water-CuO with $d_{p}=29 \mathrm{~nm}$, respectively. It is also seen that increasing the nanoparticle concentration will result in lower rates of entropy generation. Fig. 7 demonstrates that larger $\mathrm{Al}_{2} \mathrm{O}_{3}$ and smaller $\mathrm{CuO}$ nanoparticles cause higher irreversibility in the system. Additionally, the magnitude of $S_{n}$ tends to decline with increase in Reynolds number, though a slight augmentation is observed for nanofluids when the Reynolds number increases from 50 to 100. This insignificant augmentation is attributed to the intensified diffusive heat transfer and therefore higher bulk coolant temperature at $\mathrm{Re}=50$. These outcomes are in chorus with above-mentioned discussions. Total entropy generation in a device is contributed by thermal and frictional irreversibility. In order to quantize the contribution of each term in total irreversibility, the Bejan number is considered. Fig. 8 shows the value of the Bejan number as a function of Reynolds number for different nanoparticle volume-fractions, sizes and materials. It is found that the contribution of thermal entropy generation is higher than that of the fluid friction reversely correlated to the nanoparticle volume-fraction and Reynolds number. Compared to the pure-water, adding low-volume-fractions of nanoparticles and/or increasing Reynolds number cause higher frictional entropy generation. Addition of low-volume-fractions of nanoparticles in the base fluid intensifies the hydrodynamic inter-particle interactions 
causing higher viscosity. Furthermore, as a result of higher fluid viscosity at higher Reynolds numbers, due to the lower bulk coolant temperature, the frictional entropy generation is more augmented. The fluid velocity gradients also become larger at higher Reynolds numbers. Based on Eq. (26) the thermal entropy generation is related to temperature gradients. By increasing the nanoparticle concentration, the nanofluid viscosity increases, making the coolant molecules more sluggish and generating more smoother temperature pattern by stabilizing the flow field. Considering the fact that the entropy generation caused by fluid friction is far smaller than that induced by heat transfer, an exterma (minimum) does not exist in total entropy generation curve for the ranges of parameters considered here; however, an increase in the Reynolds number increases the fluid-friction-caused entropy generation and decreases heat-transfer-generated entropy .

Ultimately, Fig.9 illustrates the contours of dimensionless total entropy generation and its components at different sections of the microchannel for water-CuO flow with $\alpha=3 \%$ and $d_{p}=29.0 \mathrm{~nm}$ at $R e=150$. It is observed that employing LVGs in the microchannel changes the entropy fields. Higher entropy generation rates are expected due to fluid friction near the solid walls because of higher fluid velocity geradients (See Eq. (25)). It is seen that the core flow region and the microchannel corners are almost free of frictional entropy generation, wherein the velocity gradients extensively cancel each other out. Temperature gradients are generated in the vicinity of hot surfaces due to the large temperature difference with coolant leading to entropy generation due to heat transfer. This heat-transfer-generated entropy increases by moving towards microchannel outlet owing to thermal conductivity enhancement and higher heat absorption. The entropy generation rate caused by heat transfer is much greater than that produced by fluid friction; therefore, the total entropy generation rate is more affected by the former, increasing the Bejan number. Similar patterns of entropy generation are observed for water as well as other nanofluids studied in this work.

\section{Conclusions}

Conjugated heat transfer and single-phase laminar flow structures in a three-dimensional microchannel equipped with longitudinal vortex generators were numerically investigated. Water- $\mathrm{Al}_{2} \mathrm{O}_{3}$ and water- $\mathrm{CuO}$ nanofluids with different nanoparticle volume-fractions and sizes were compared to pure-water as working fluids. The results of present numerical simulations under steady-state assumption were in good agreement with the available experimental and numerical data leading to the following major findings and conclusions.

Using nanofluids as coolant can enhance heat transfer performance of the rectangular microchannels heat sinks equipped with LVGs with a larger pressure drop penalty. In addition, nanofluids with higher nanoparticle concentrations although again cost higher pressure drop, result in greater heat transfer enhancement. Decreasing the size of $\mathrm{Al}_{2} \mathrm{O}_{3}$ nanoparticles enhances the heat transfer performance and also imposes larger pressure drop; while, on the other hand, heat transfer performance and the Fanning friction factor are augmented by increasing the diameter of $\mathrm{CuO}$ nanoparticles. Also for comparable nanoparticle size and volume-fraction, water- $\mathrm{CuO}$ show higher heat transfer performance and pressure drop compared to water- $\mathrm{Al}_{2} \mathrm{O}_{3}$ for the all range of Reynolds number studied in this paper. A notable increase in overall efficiency is found by using 
nanofluids as coolant; where this enhancement will boost by increasing the nanoparticle volume-fraction. The flows with low Reynolds number are the best operational regime for rectangular microchannels heat sinks with LVGs using nanofluids as working fluid. The overall efficiency of the considered microchannel increases by increasing the $\mathrm{CuO}$ nanoparticle diameter and decreasing the size of $\mathrm{Al}_{2} \mathrm{O}_{3}$ nanoparticles. Among all the cases studied in this paper, water- $\mathrm{CuO}$ nanofluid with $d_{p}=29$ and highest nanoparticle volume-fraction (i.e. $\varphi=3.0 \%$ ) shows the best overall efficiency. Based on second law analysis, nanofluids are an excellent option for heat transfer applications and could enhance the thermodynamic performance of the device. 


\section{References}

1. Tuckerman, D.B. and R. Pease, High-performance heat sinking for VLSI. Electron Device Letters, IEEE, 1981. 2(5): p. 126-129.

2. Morini, G.L., Single-phase convective heat transfer in microchannels: a review of experimental results. International Journal of Thermal Sciences, 2004. 43(7): p. 631-651.

3. Agostini, B., et al., State of the art of high heat flux cooling technologies. Heat Transfer Engineering, 2007. 28(4): p. 258-281.

4. Adham, A.M., N. Mohd-Ghazali, and R. Ahmad, Thermal and hydrodynamic analysis of microchannel heat sinks: A review. Renewable and Sustainable Energy Reviews, 2013. 21: p. 614-622.

5. Li, Z., W.-Q. Tao, and Y.-L. He, A numerical study of laminar convective heat transfer in microchannel with non-circular cross-section. International Journal of Thermal Sciences, 2006. 45(12): p. $1140-1148$.

6. Koyuncuoğlu, A., et al., Heat transfer and pressure drop experiments on CMOS compatible microchannel heat sinks for monolithic chip cooling applications. International Journal of Thermal Sciences, 2012. 56: p. 77-85.

7. Masuda, H., et al., Alteration of thermal conductivity and viscosity of liquid by dispersing ultrafine particles. Netsu Bussei, 1993. 7(4): p. 227-233.

8. Chol, S., Enhancing thermal conductivity of fluids with nanoparticles. ASME-PublicationsFed, 1995. 231: p. 99-106.

9. Eastman, J., et al. Enhanced thermal conductivity through the development of nanofluids. in MRS Proceedings. 1996. Cambridge Univ Press.

10. Wang, X., X. Xu, and S.U. S. Choi, Thermal conductivity of nanoparticle-fluid mixture. Journal of thermophysics and heat transfer, 1999. 13(4): p. 474-480.

11. Murshed, S., K. Leong, and C. Yang, Enhanced thermal conductivity of TiO 2-water based nanofluids. International Journal of Thermal Sciences, 2005. 44(4): p. 367-373.

12. Li, C.H. and G. Peterson, Experimental investigation of temperature and volume fraction variations on the effective thermal conductivity of nanoparticle suspensions (nanofluids). Journal of Applied Physics, 2006. 99(8): p. 084314.

13. Kumaresan, V. and R. Velraj, Experimental investigation of the thermo-physical properties of water-ethylene glycol mixture based CNT nanofluids. Thermochimica Acta, 2012. 545: p. 180-186.

14. Shylaja, A., et al., Preparation and Thermo-Physical Properties of Fe2O3-Propylene Glycol Nanofluids. Journal of Nanoscience and Nanotechnology, 2015. 15(2): p. 1653-1659.

15. Sadeghinezhad, E., et al., An experimental and numerical investigation of heat transfer enhancement for graphene nanoplatelets nanofluids in turbulent flow conditions. International Journal of Heat and Mass Transfer, 2015. 81: p. 41-51.

16. Ma, L., et al., Viscosity and Thermal Conductivity of Stable Graphite Suspensions Near Percolation. Nano letters, 2015.

17. Godson, L., et al., Enhancement of heat transfer using nanofluids-an overview. Renewable and Sustainable Energy Reviews, 2010. 14(2): p. 629-641.

18. Sarkar, J., A critical review on convective heat transfer correlations of nanofluids. Renewable and Sustainable Energy Reviews, 2011. 15(6): p. 3271-3277. 
19. Mahbubul, I., R. Saidur, and M. Amalina, Latest developments on the viscosity of nanofluids. International Journal of Heat and Mass Transfer, 2012. 55(4): p. 874-885.

20. Hussein, A.M., et al., A review of forced convection heat transfer enhancement and hydrodynamic characteristics of a nanofluid. Renewable and Sustainable Energy Reviews, 2014. 29: p. 734-743.

21. Sarkar, J., P. Ghosh, and A. Adil, A review on hybrid nanofluids: Recent research, development and applications. Renewable and Sustainable Energy Reviews, 2015. 43: p. 164-177.

22. Pang, C., J.W. Lee, and Y.T. Kang, Review on combined heat and mass transfer characteristics in nanofluids. International Journal of Thermal Sciences, 2015. 87: p. 49-67.

23. Kalteh, M., et al., Eulerian-Eulerian two-phase numerical simulation of nanofluid laminar forced convection in a microchannel. International journal of heat and fluid flow, 2011. 32(1): p. 107116.

24. Kalteh, M., et al., Experimental and numerical investigation of nanofluid forced convection inside a wide microchannel heat sink. Applied Thermal Engineering, 2012. 36: p. 260-268.

25. Sohel, M., et al., Investigating the heat transfer performance and thermophysical properties of nanofluids in a circular micro-channel. International Communications in Heat and Mass Transfer, 2013. 42: p. $75-81$.

26. Mital, M., Analytical analysis of heat transfer and pumping power of laminar nanofluid developing flow in microchannels. Applied Thermal Engineering, 2013. 50(1): p. 429-436.

27. Rimbault, B., C.T. Nguyen, and N. Galanis, Experimental investigation of $\mathrm{CuO}$-water nanofluid flow and heat transfer inside a microchannel heat sink. International Journal of Thermal Sciences, 2014. 84: p. 275-292.

28. Nimmagadda, R. and K. Venkatasubbaiah, Conjugate heat transfer analysis of micro-channel using novel hybrid nanofluids $\left(\mathrm{Al}_{2} \mathrm{O}_{3}+\mathrm{Ag} /\right.$ Water $)$. European Journal of Mechanics-B/Fluids, 2015. 52: p. 19-27.

29. Daniel Makinde, O., Effects of viscous dissipation and Newtonian heating on boundary-layer flow of nanofluids over a flat plate. International Journal of Numerical Methods for Heat \& Fluid Flow, 2013. 23(8): p. 1291-1303.

30. Makinde, O. D., W. A. Khan, and A. Aziz, On inherent irreversibility in Sakiadis flow of nanofluids. International Journal of Exergy, 2013. 13(2): p. 159-174.

31. Khamis, S., D. O. Makinde, and Y. Nkansah-Gyekye, Unsteady flow of variable viscosity Cuwater and Al2O3-water nanofluids in a porous pipe with buoyancy force. International Journal of Numerical Methods for Heat \& Fluid Flow, 2015. 25(7): p. 1638-1657.

32. Bergles, A.E., Recent developments in enhanced heat transfer. Heat and mass transfer, 2011. 47(8): p. 1001-1008.

33. Webb, R.L. and N.-H. Kim, Principles of enhanced heat transfer. 2nd ed. 2005, Boca Raton: Taylor \& Francis. xxii, 795 p.

34. Johnson, T.R. and P.N. Joubert, The influence of vortex generators on the drag and heat transfer from a circular cylinder normal to an airstream. Journal of Heat Transfer, 1969. 91: p. 91.

35. Sohankar, A., Heat transfer augmentation in a rectangular channel with a vee-shaped vortex generator. International journal of heat and fluid flow, 2007. 28(2): p. 306-317.

36. Tian, L.-T., et al., Numerical study of fluid flow and heat transfer in a flat-plate channel with longitudinal vortex generators by applying field synergy principle analysis. International Communications in Heat and Mass Transfer, 2009. 36(2): p. 111-120. 
37. Ebrahimi, A. and S. Kheradmand, Numerical Simulation of Performance Augmentation in a Plate Fin Heat Exchanger Using Winglet Type Vortex Generators. International Journal of Mechanical Engineering and Mechatronics, 2012. 1(1): p. 109-121.

38. Fiebig, M., Embedded vortices in internal flow: heat transfer and pressure loss enhancement. International Journal of Heat and Fluid Flow, 1995. 16(5): p. 376-388.

39. Ahmed, H., H.A. Mohammed, and M. Yusoff, An overview on heat transfer augmentation using vortex generators and nanofluids: Approaches and applications. Renewable and Sustainable Energy Reviews, 2012. 16(8): p. 5951-5993.

40. Liu, C., et al., Experimental investigations on liquid flow and heat transfer in rectangular microchannel with longitudinal vortex generators. International Journal of Heat and Mass Transfer, 2011. 54(13): p. 3069-3080.

41. Lan, J., Y. Xie, and D. Zhang, Flow and Heat Transfer in Microchannels With Dimples and Protrusions. Journal of heat transfer, 2012. 134(2).

42. Mirzaee, H., et al., Heat Transfer Enhancement in Microchannels using an Elastic Vortex Generator. Journal of Enhanced Heat Transfer, 2012. 19(3).

43. Chen, C., et al., A study on fluid flow and heat transfer in rectangular microchannels with various longitudinal vortex generators. International Journal of Heat and Mass Transfer, 2014. 69: p. 203-214.

44. $\mathrm{Li}, \mathrm{P} ., \mathrm{D}$. Zhang, and Y. Xie, Heat transfer and flow analysis of $\mathrm{Al}_{2} \mathrm{O}_{3}$-water nanofluids in microchannel with dimple and protrusion. International Journal of Heat and Mass Transfer, 2014. 73: p. 456-467.

45. Ebrahimi, A., E. Roohi, and S. Kheradmand, Numerical study of liquid flow and heat transfer in rectangular microchannel with longitudinal vortex generators. Applied Thermal Engineering, 2015. 78: p. $576-583$.

46. Kandlikar, S.G. and W.J. Grande, Evolution of Microchannel Flow Passages--Thermohydraulic Performance and Fabrication Technology. Heat transfer engineering, 2003. 24(1): p. 3-17.

47. Seyf, H.R. and M. Feizbakhshi, Computational analysis of nanofluid effects on convective heat transfer enhancement of micro-pin-fin heat sinks. International Journal of Thermal Sciences, 2012. 58: p. $168-179$.

48. Yue, Y., S.K. Mohammadian, and Y. Zhang, Analysis of performances of a manifold microchannel heat sink with nanofluids. International Journal of Thermal Sciences, 2015. 89: p. 305313.

49. Ferrouillat, S., et al., Intensification of heat-transfer and mixing in multifunctional heat exchangers by artificially generated streamwise vorticity. Applied thermal engineering, 2006. 26(16): p. $1820-1829$.

50. Incropera, F.P., Fundamentals of heat and mass transfer. 2011: John Wiley \& Sons.

51. Etminan-Farooji, V., et al., Unconfined laminar nanofluid flow and heat transfer around a square cylinder. International Journal of Heat and Mass Transfer, 2012. 55(5): p. 1475-1485.

52. Glassbrenner, C. and G.A. Slack, Thermal conductivity of silicon and germanium from $3 \mathrm{~K}$ to the melting point. Physical Review, 1964. 134(4A): p. A1058.

53. Pak, B.C. and Y.I. Cho, Hydrodynamic and heat transfer study of dispersed fluids with submicron metallic oxide particles. Experimental Heat Transfer an International Journal, 1998. 11(2): p. 151-170. 
54. Xuan, Y. and W. Roetzel, Conceptions for heat transfer correlation of nanofluids. International Journal of heat and Mass transfer, 2000. 43(19): p. 3701-3707.

55. Koo, J. and C. Kleinstreuer, A new thermal conductivity model for nanofluids. Journal of Nanoparticle Research, 2004. 6(6): p. 577-588.

56. $\mathrm{Li}, \mathrm{J}$. and C. Kleinstreuer, Thermal performance of nanofluid flow in microchannels. International Journal of Heat and Fluid Flow, 2008. 29(4): p. 1221-1232.

57. Hamilton, R. and O. Crosser, Thermal conductivity of heterogeneous two-component systems. Industrial \& Engineering chemistry fundamentals, 1962. 1(3): p. 187-191.

58. Li, J., Computational Analysis of Nanofluid Flow in Microchannels with Applications to Micro-heat Sinks and Bio-MEMS. 2008: ProQuest.

59. Nandi, T.K. and H. Chattopadhyay, Numerical investigations of developing flow and heat transfer in raccoon type microchannels under inlet pulsation. International Communications in Heat and Mass Transfer, 2014. 56: p. 37-41.

60. Zhai, Y., et al., Heat transfer in the microchannels with fan-shaped reentrant cavities and different ribs based on field synergy principle and entropy generation analysis. International Journal of Heat and Mass Transfer, 2014. 68: p. 224-233.

61. Ebrahimi, A. and E. Roohi, Numerical Study of Flow Patterns and Heat Transfer in Mini Twisted Oval Tubes. International Journal of Modern Physics C, $2015.26(12)$. doi:10.1142/S0129183115501405

62. Li, J., and C. Kleinstreuer, Entropy Generation Analysis for Nanofluid Flow in Microchannels. Journal of Heat Transfer, 2010. 132: p. 122401.

63. Lee, J. and I. Mudawar, Assessment of the effectiveness of nanofluids for single-phase and two-phase heat transfer in micro-channels. International Journal of Heat and Mass Transfer, 2007. 50(3): p. 452-463.

64. Ghale, Z.Y., M. Haghshenasfard, and M.N. Esfahany, Investigation of nanofluids heat transfer in a ribbed microchannel heat sink using single-phase and multiphase CFD models. International Communications in Heat and Mass Transfer, 2015. 68: p. 122-129.

65. Lai, W., S. Vinod, P. Phelan, R. Prasher, Convective heat transfer for water-based alumina nanofluids in a single 1.02-mm tube. Journal of Heat Transfer, 2009. 131(11): p. 112401.

66. Akbari, M., N. Galanis, A. Behzadmehr, Comparative assessment of single and two-phase models for numerical studies of nanofluid turbulent forced convection. International Journal of Heat and Fluid Flow, 2012. 37: p. 136-146.

67. Behroyan, I., Sh M. Vanaki, P. Ganesan, and R. Saidur. A comprehensive comparison of various CFD models for convective heat transfer of $\mathrm{Al}_{2} \mathrm{O}_{3}$ nanofluid inside a heated tube. International Communications in Heat and Mass Transfer, 2016. 70: p. 27-37.

68. Hanafizadeh, P., M. Ashjaee, M. Goharkhah, K. Montazeri, and M. Akram. The comparative study of single and two-phase models for magnetite nanofluid forced convection in a tube. International Communications in Heat and Mass Transfer, 2015. 65: p. 58-70.

69. Kandelousi, M.S., KKL correlation for simulation of nanofluid flow and heat transfer in a permeable channel. Physics Letters A, 2014. 378(45): p. 3331-3339.

70. Keblinski, P., J.A. Eastman, and D.G. Cahill, Nanofluids for thermal transport. Materials today, 2005. 8(6): p. 36-44.

71. Pang, C., J.-Y. Jung, and Y.T. Kang, Aggregation based model for heat conduction mechanism in nanofluids. International Journal of Heat and Mass Transfer, 2014. 72: p. 392-399. 
72. Pang, C., J.W. Lee, and Y.T. Kang, Enhanced thermal conductivity of nanofluids by nanoconvection and percolation network. Heat and Mass Transfer, 2015: p. 1-10.

73. Hunter, R.J., Foundations of colloid science. 2001.

74. Prasher, R., P.E. Phelan, and P. Bhattacharya, Effect of aggregation kinetics on the thermal conductivity of nanoscale colloidal solutions (nanofluid). Nano Letters, 2006. 6(7): p. 1529-1534. 


\section{List of tables}

Table 1- Characteristic dimensions of the microchannel and the LVGs located inside it.

Table 2- Thermophysical properties of nanoparticles, pure-water and silicon.

Table 3- Results of grid independence tests. (Working fluid: Pure-water, $R e=250$ ) 


\section{List of figures}

Fig. 1- Schematic diagrams of the physical model and (a) associated geometrical parameters (b) computational domain with the specified vertices to set boundary conditions.

Fig. 2- Comparision of present simulation results with available experimental $[63,65]$ and numerical [64] data sets. (a) Heat transfer coefficient versus Reynolds numbers. (b) Fanning friction factor versus Reynolds numbers. (c) Local heat transfer coefficients in the thermaldeveloping region of tube.

Fig. 3- Variations of Nusselt number and Fanning friction factor with Reynolds number for nanofluids containing $\mathrm{Al}_{2} \mathrm{O}_{3}$ or $\mathrm{CuO}$ nanoparticles with different concentrations. $\left(d_{p}=29 \mathrm{~nm}\right)$

Fig. 4- Effects of nanoparticle mean diameter on Nusselt number and Fanning friction factor for $3.0 \%$ volume-fraction of $\mathrm{Al}_{2} \mathrm{O}_{3}$ or $\mathrm{CuO}$ nanoparticles.

Fig. 5- The effects of different nanofluid characteristics on flow and thermal fields for $R e=150$. (the plane locates at $\mathrm{y} / \mathrm{H}=0.5$ )

Fig. 6- The overall efficiency of the microchannels with LVGs for water-CuO and water- $\mathrm{Al}_{2} \mathrm{O}_{3}$ nanofluids. (a) Effects of nanoparticle volume-fraction; (b) Effects of nanoparticle size.

Fig. 7- The dimensionless entropy generation $\left(S_{n}\right)$ as a function of Reynolds number for nanofluids containing $\mathrm{Al}_{2} \mathrm{O}_{3}$ or $\mathrm{CuO}$ nanoparticles of different sizes with various concentrations.

Fig. 8- Variations of Bejan number versus Reynolds number for nanofluids containing $\mathrm{Al}_{2} \mathrm{O}_{3}$ or $\mathrm{CuO}$ nanoparticles of different sizes with various concentrations.

Fig. 9- Contours of dimensionless entropy generation due to fluid friction (top), heat transfer (middle) and both sources (bottom) for water-CuO flow with $\alpha=3 \%$ and $d_{p}=29.0 \mathrm{~nm}$ at $R e=150$. 
Table 1- Characteristic dimensions of the microchannel and the LVGs located inside it.

\begin{tabular}{lllll}
\hline Geometric parameter & Value & & Geometric parameter & Value \\
\cline { 1 - 2 } & $100 \mu \mathrm{m}$ & & $\mathrm{L}_{\text {in }}$ & $25 \mathrm{H}$ \\
$\beta$ & $30^{\circ}$ & $\mathrm{L}_{\mathrm{ht}}$ & $100 \mathrm{H}$ \\
$\mathrm{d}_{\mathrm{LVG}}$ & $16 \mathrm{H}$ & & $\mathrm{L}_{\text {out }}$ & $50 \mathrm{H}$ \\
$\mathrm{W}_{\mathrm{LVG}}$ & $0.1 \mathrm{H}$ & $\mathrm{L}_{1}$ & $10 \mathrm{H}$ \\
$\mathrm{L}_{\mathrm{LVG}}$ & $1.4 \mathrm{H}$ & & $\mathrm{W}$ & $4 \mathrm{H}$ \\
$\mathrm{a}$ & $0.8 \mathrm{H}$ & & $\mathrm{D}_{\mathrm{h}}$ & $160 \mu \mathrm{m}$ \\
\hline
\end{tabular}


Table 2- Thermophysical properties of nanoparticles, pure-water and silicon. $\mathrm{Al}_{2} \mathrm{O}_{3}[50] \quad \mathrm{CuO}$ [51] $\quad$ Silicon [52] $\quad$ Pure-water [48]

\begin{tabular}{lllll}
\hline$\mu(\mathrm{Pa} . \mathrm{s})$ & & & & $2.761 \times 10^{-6} \exp \left(\frac{1713}{T}\right)$ \\
$k(\mathrm{~W} / \mathrm{m} \mathrm{K})$ & 36 & 76.5 & $290-0.4 T$ & $0.6\left(1+4.167 \times 10^{-5} T\right)$ \\
$c_{p}(\mathrm{~J} / \mathrm{kg} \mathrm{K})$ & 765 & 535.6 & $390+0.9 T$ & 4180 \\
$\rho\left(\mathrm{kg} / \mathrm{m}^{3}\right)$ & 3970 & 6350 & 2330 & 1000 \\
\hline
\end{tabular}


Table 3- Results of grid independence tests. (Working fluid: Pure-water, Re=250)

\begin{tabular}{lllll}
\hline Number of cells & $N u$ & $\%$ Diff $N u$ & $f$ & \% Diff $f$ \\
\hline 300000 & 9.2343 & - & 37.3290 & - \\
500000 & 7.5793 & -17.92 & 30.3313 & -18.75 \\
1000000 & 7.5466 & -0.43 & 30.1890 & -0.47 \\
1500000 & 7.5646 & 0.24 & 30.2155 & 0.09 \\
\hline
\end{tabular}




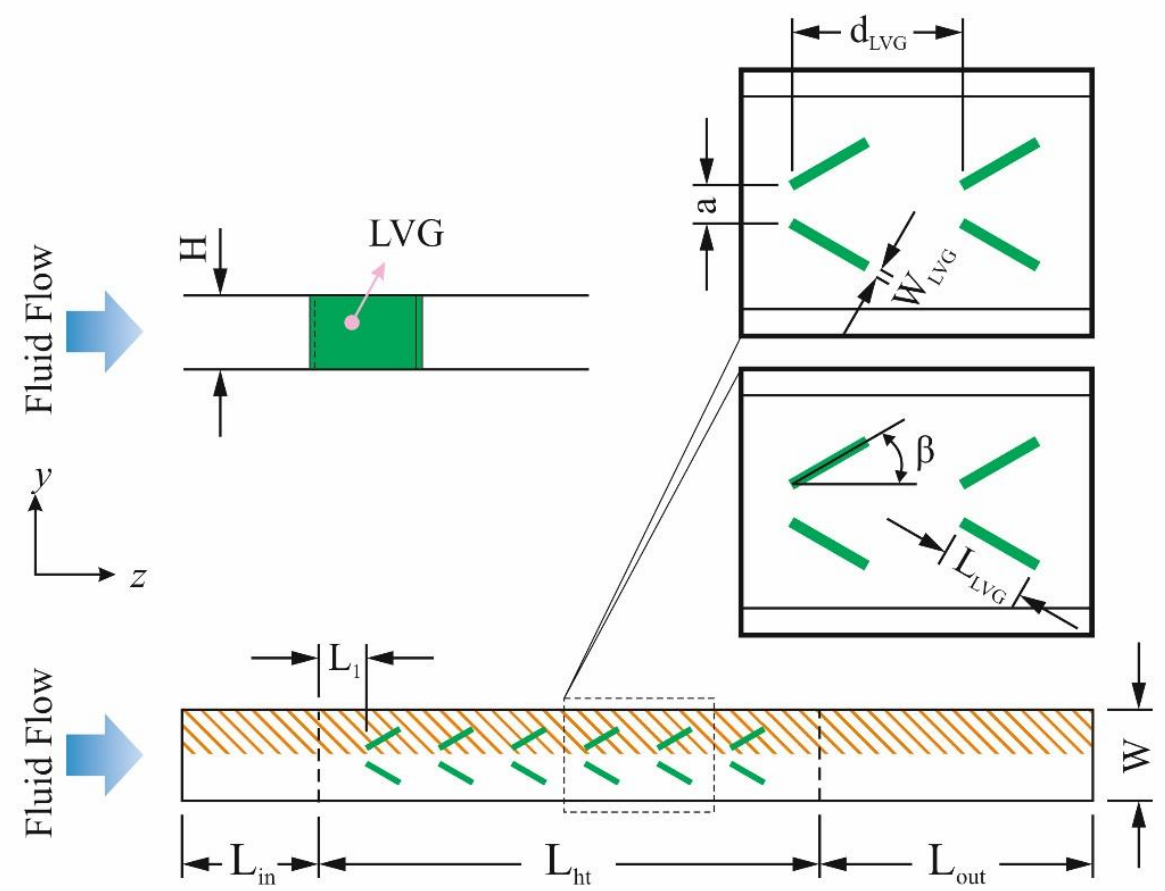

$\stackrel{x}{\longrightarrow} z$

(a)

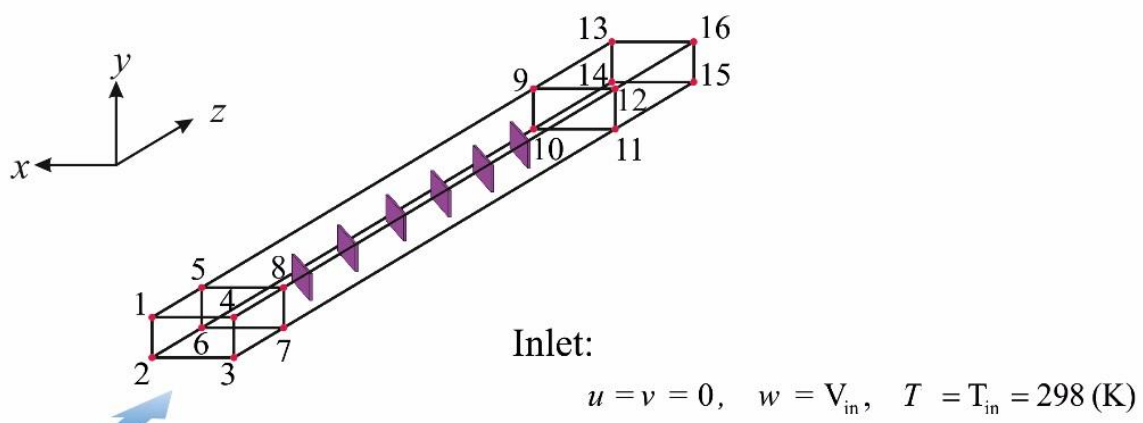

Fluid Flow

Outlet:

(b)

$$
\frac{\partial u}{\partial z}=\frac{\partial v}{\partial z}=\frac{\partial w}{\partial z}=0, \quad \frac{\partial T}{\partial z}=0
$$

Symmetry plane:

$$
\frac{\partial v}{\partial x}=\frac{\partial w}{\partial x}=0, \quad \frac{\partial T}{\partial x}=\frac{\partial T_{s}}{\partial x}=0, \quad u=0
$$

Heated wall:

$$
u=v=w=0, \quad q_{w}^{\prime \prime}=-k \frac{\partial T}{\partial \mathbf{n}}
$$

Adiabatic walls:

$$
u=v=w=0, \quad \frac{\partial T}{\partial y}=\frac{\partial T_{s}}{\partial y}=0
$$

Fig. 1- Schematic diagrams of the physical model and (a) associated geometrical parameters (b) computational domain with the specified vertices to set boundary conditions. 

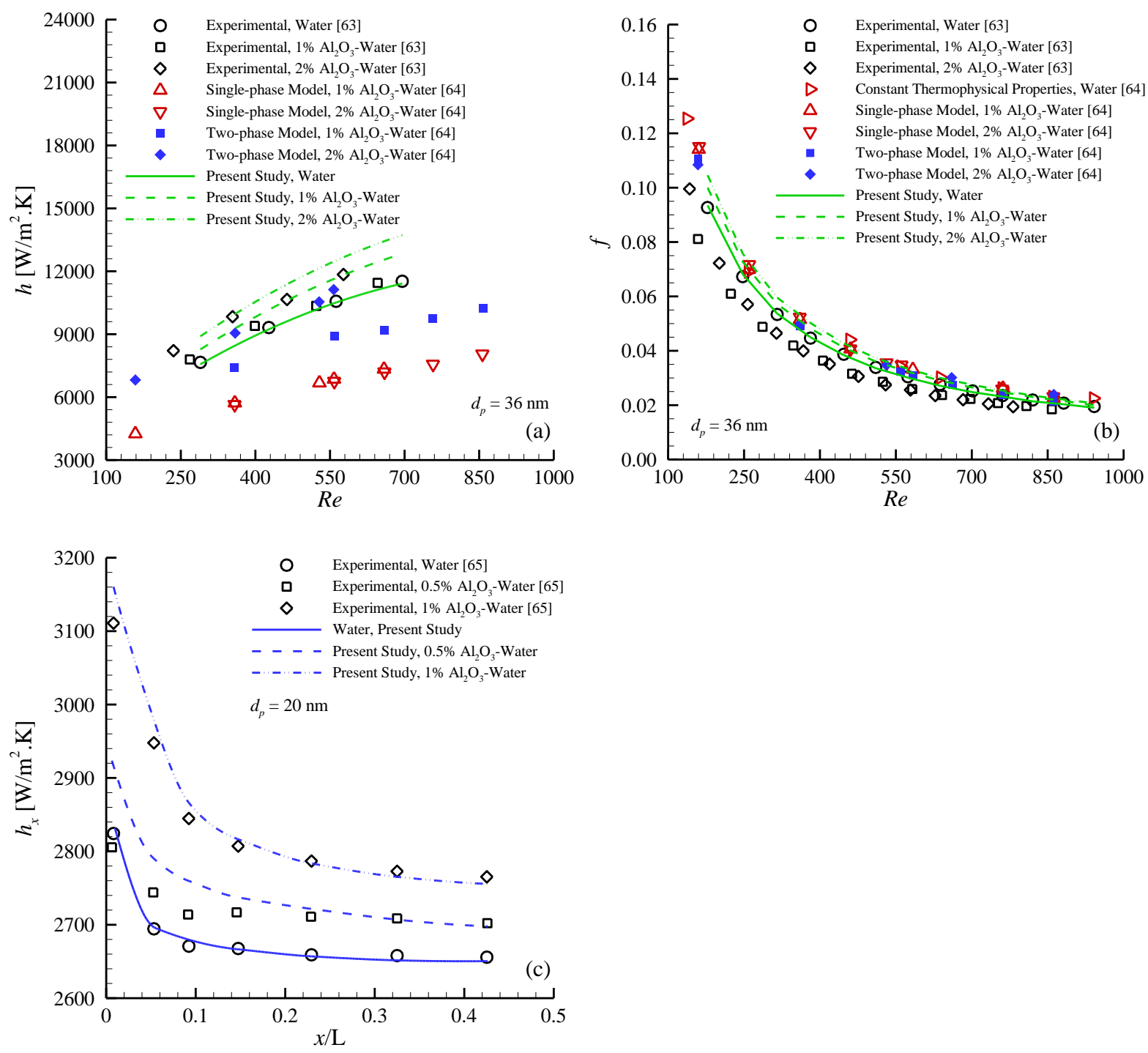

Fig. 2- Comparision of present simulation results with available experimental $[63,65]$ and numerical [64] data sets. (a) Heat transfer coefficient versus Reynolds numbers. (b) Fanning friction factor versus Reynolds numbers. (c) Local heat transfer coefficients in the thermaldeveloping region of tube. 


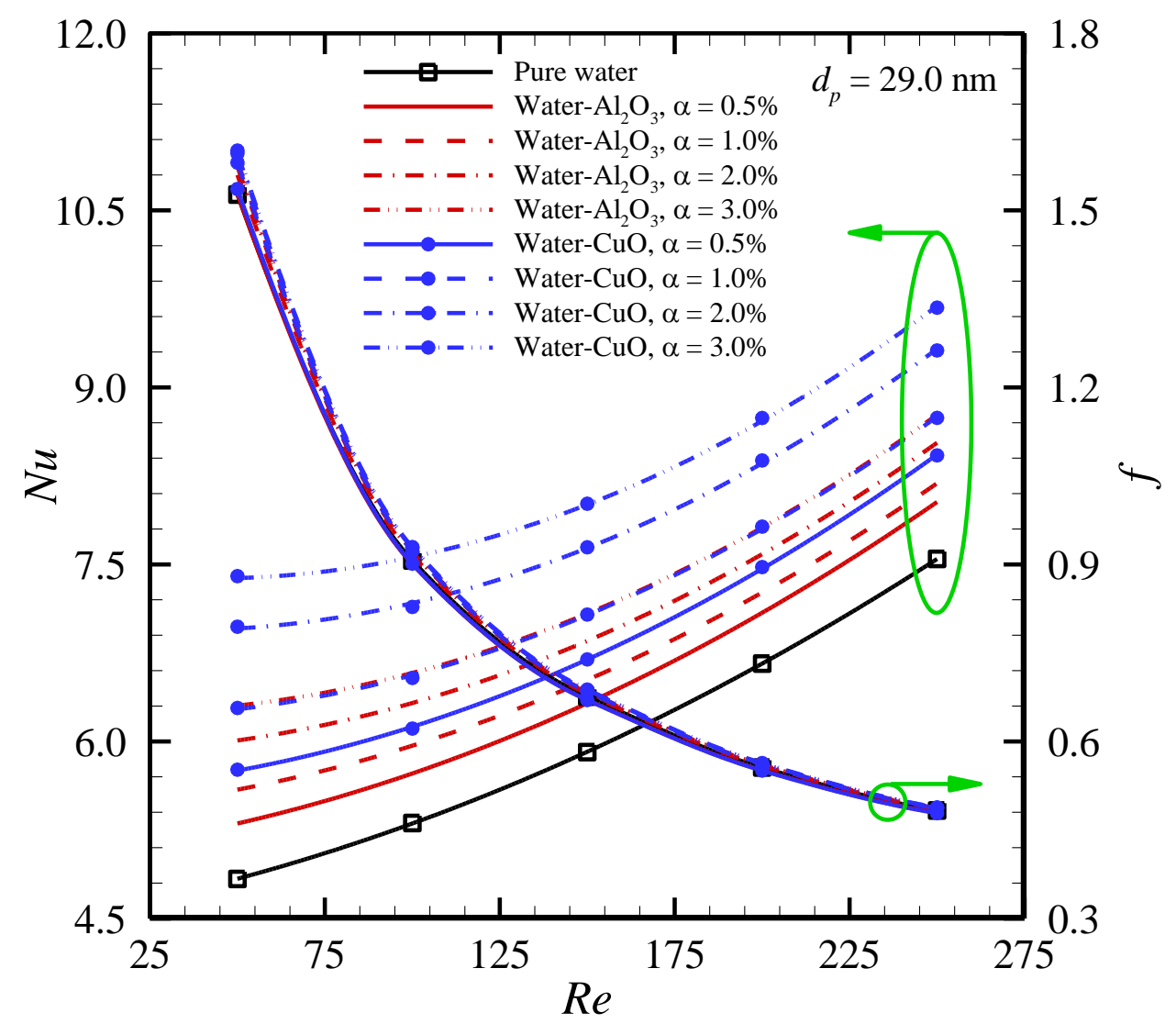

Fig. 3- Variations of Nusselt number and Fanning friction factor with Reynolds number for nanofluids containing $\mathrm{Al}_{2} \mathrm{O}_{3}$ or $\mathrm{CuO}$ nanoparticles with different concentrations. $\left(d_{p}=29 \mathrm{~nm}\right)$ 


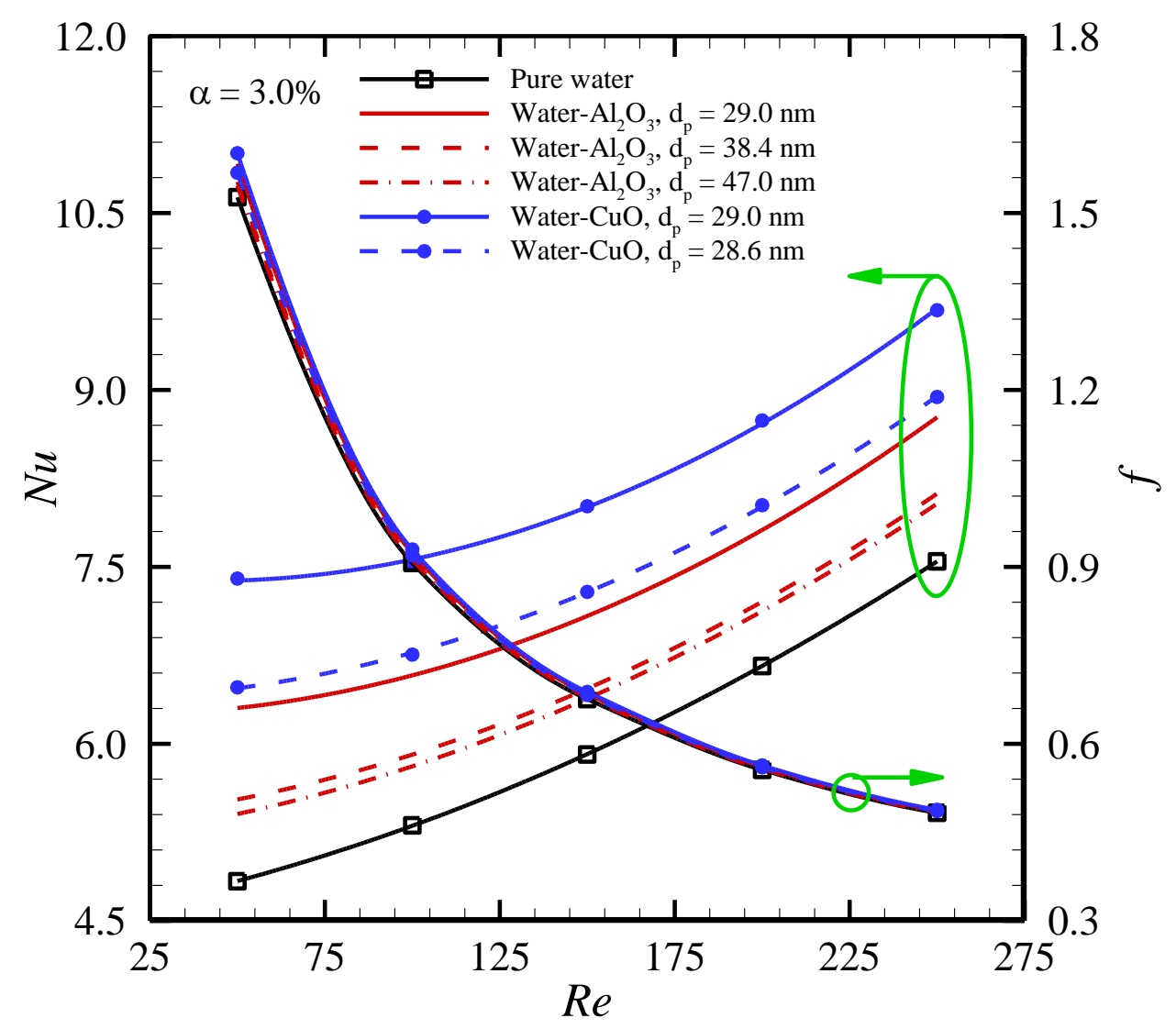

Fig. 4- Effects of nanoparticle mean diameter on Nusselt number and Fanning friction factor for $3.0 \%$ volume-fraction of $\mathrm{Al}_{2} \mathrm{O}_{3}$ or $\mathrm{CuO}$ nanoparticles. 


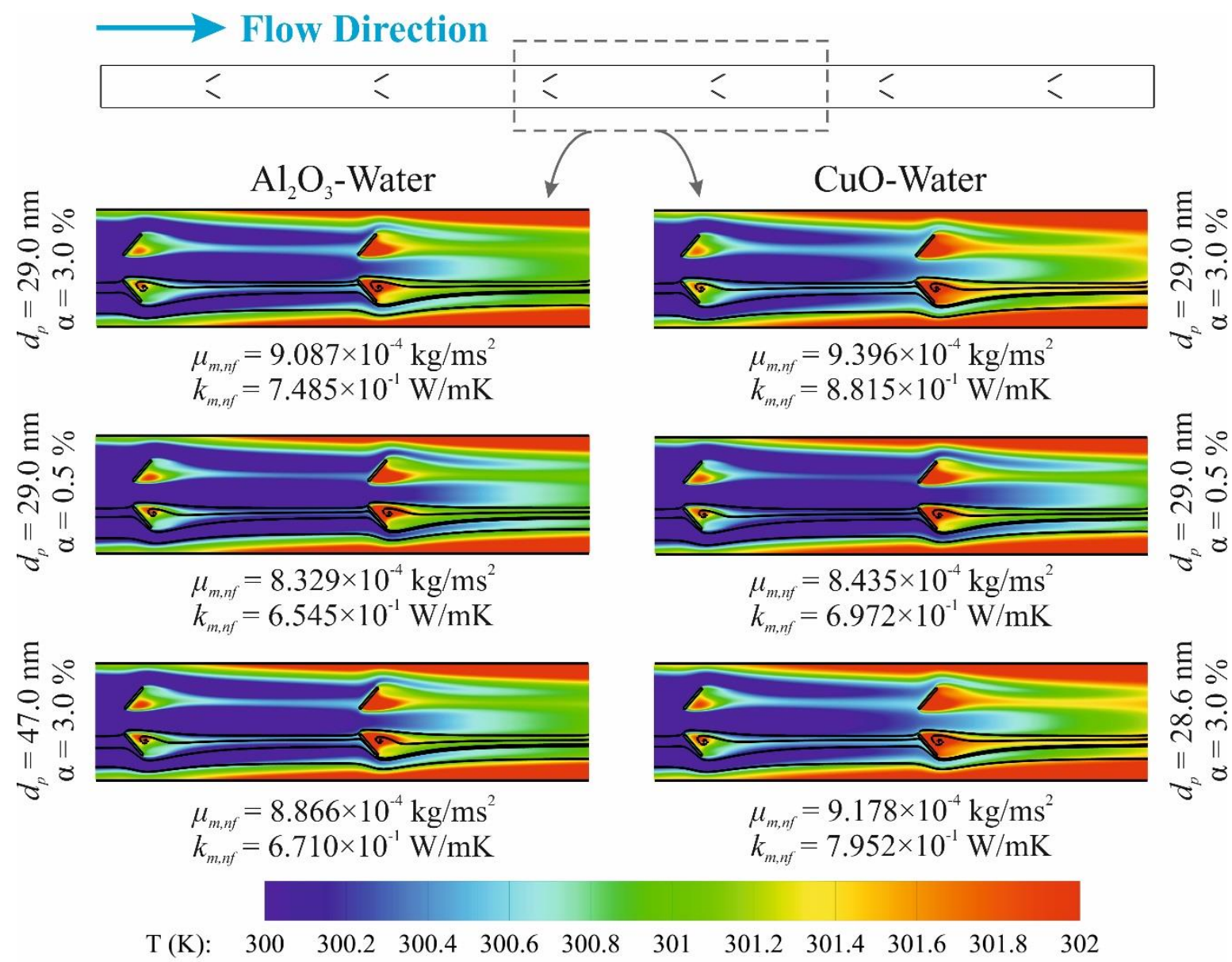

Fig. 5- The effects of different nanofluid characteristics on flow and thermal fields for $R e=150$. (the plane locates at $\mathrm{y} / \mathrm{H}=0.5$ ) 

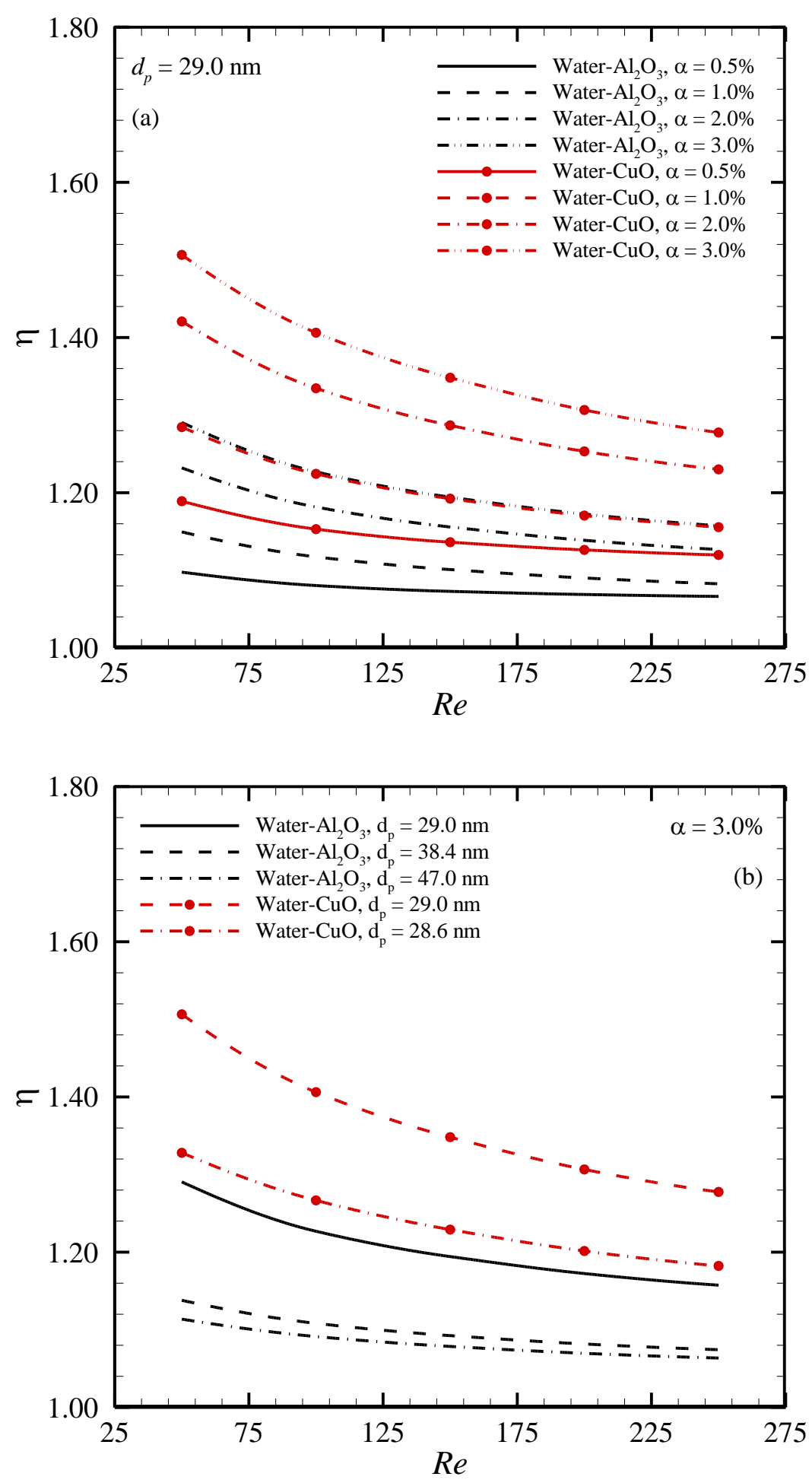

Fig. 6- The overall efficiency of the microchannels with LVGs for water-CuO and water- $\mathrm{Al}_{2} \mathrm{O}_{3}$ nanofluids. (a) Effects of nanoparticle volume-fraction; (b) Effects of nanoparticle size. 


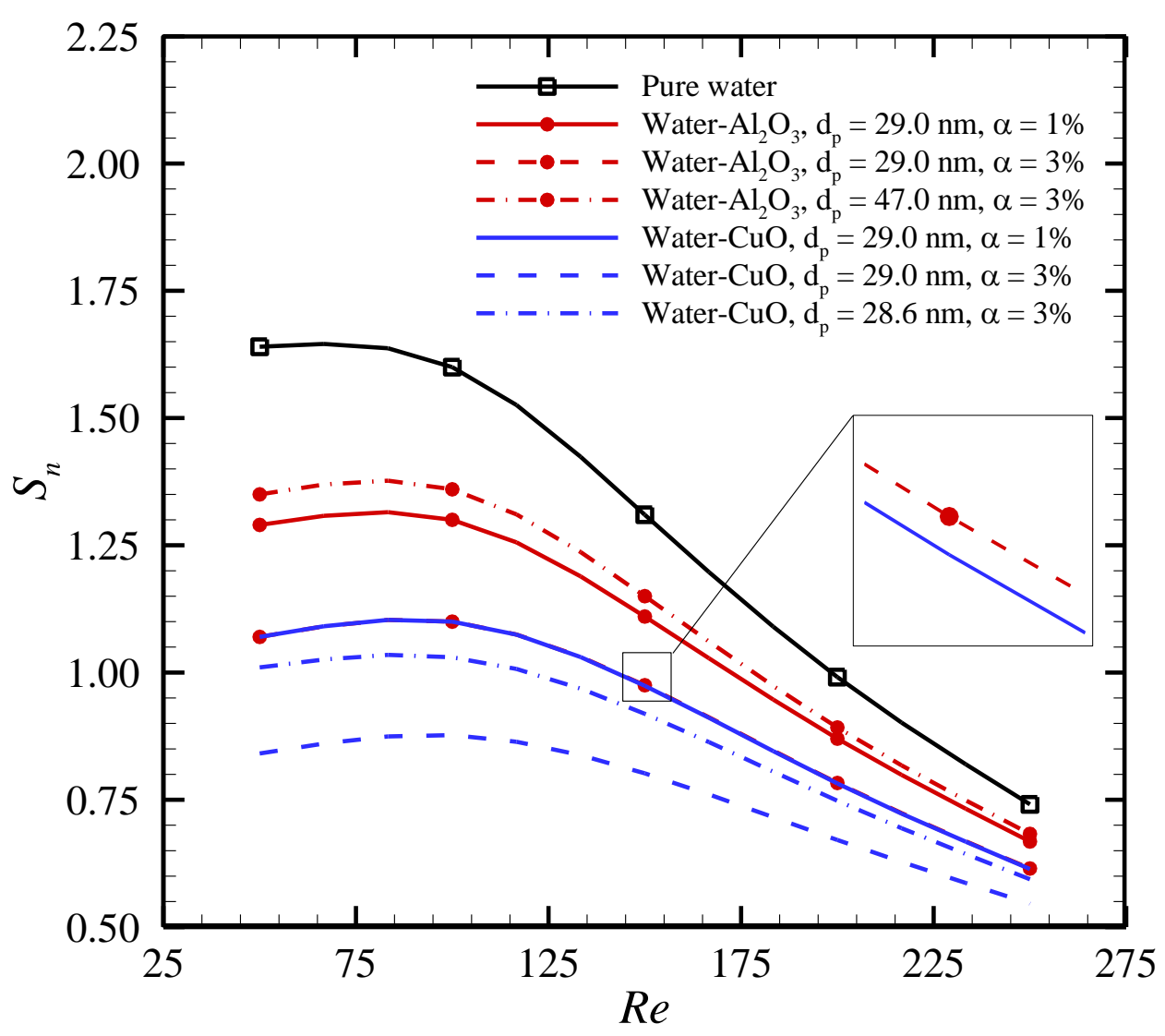

Fig. 7- The dimensionless entropy generation $\left(S_{n}\right)$ as a function of Reynolds number for nanofluids containing $\mathrm{Al}_{2} \mathrm{O}_{3}$ or $\mathrm{CuO}$ nanoparticles of different sizes with various concentrations. 


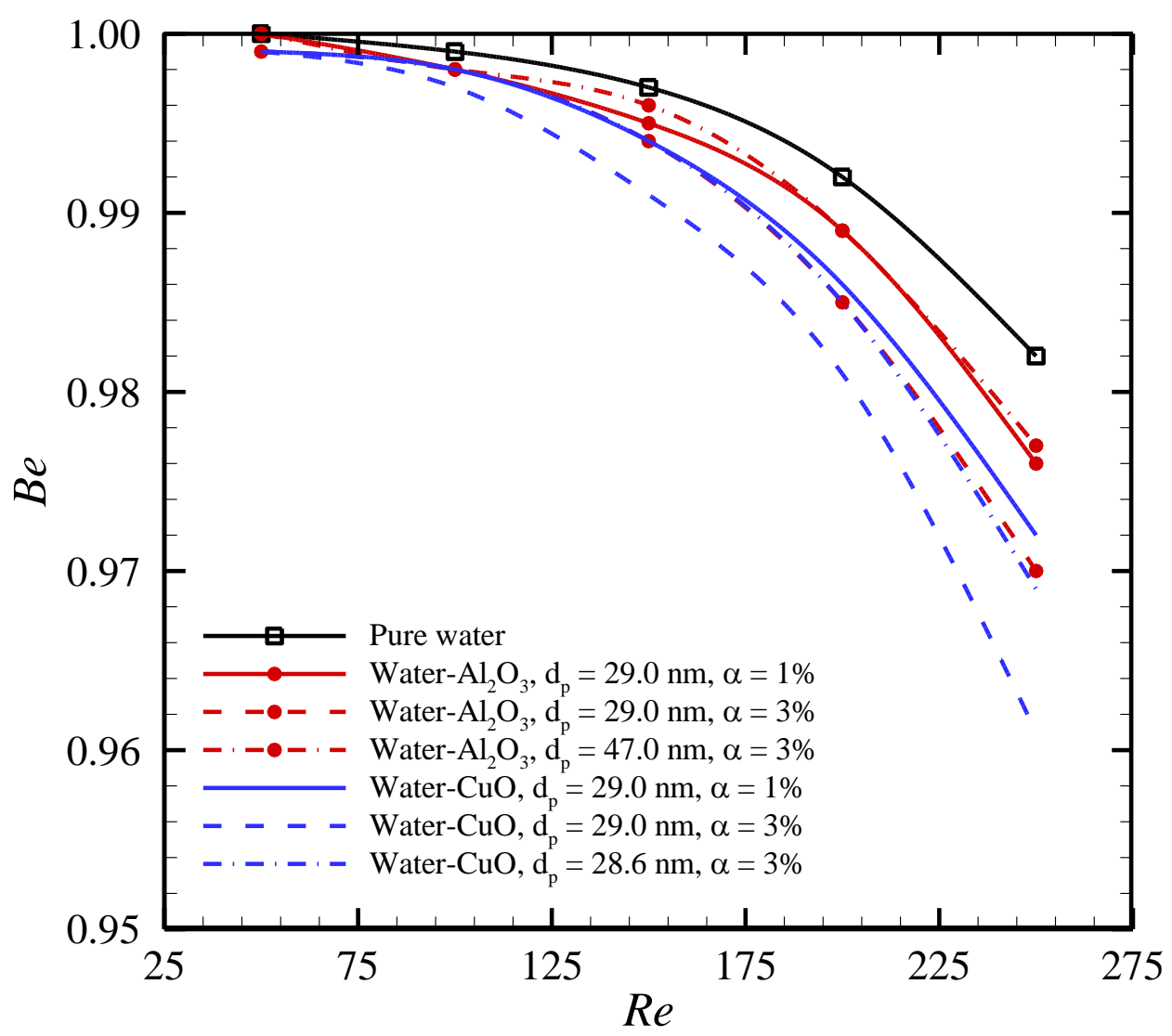

Fig. 8- Variations of Bejan number versus Reynolds number for nanofluids containing $\mathrm{Al}_{2} \mathrm{O}_{3}$ or $\mathrm{CuO}$ nanoparticles of different sizes with various concentrations. 


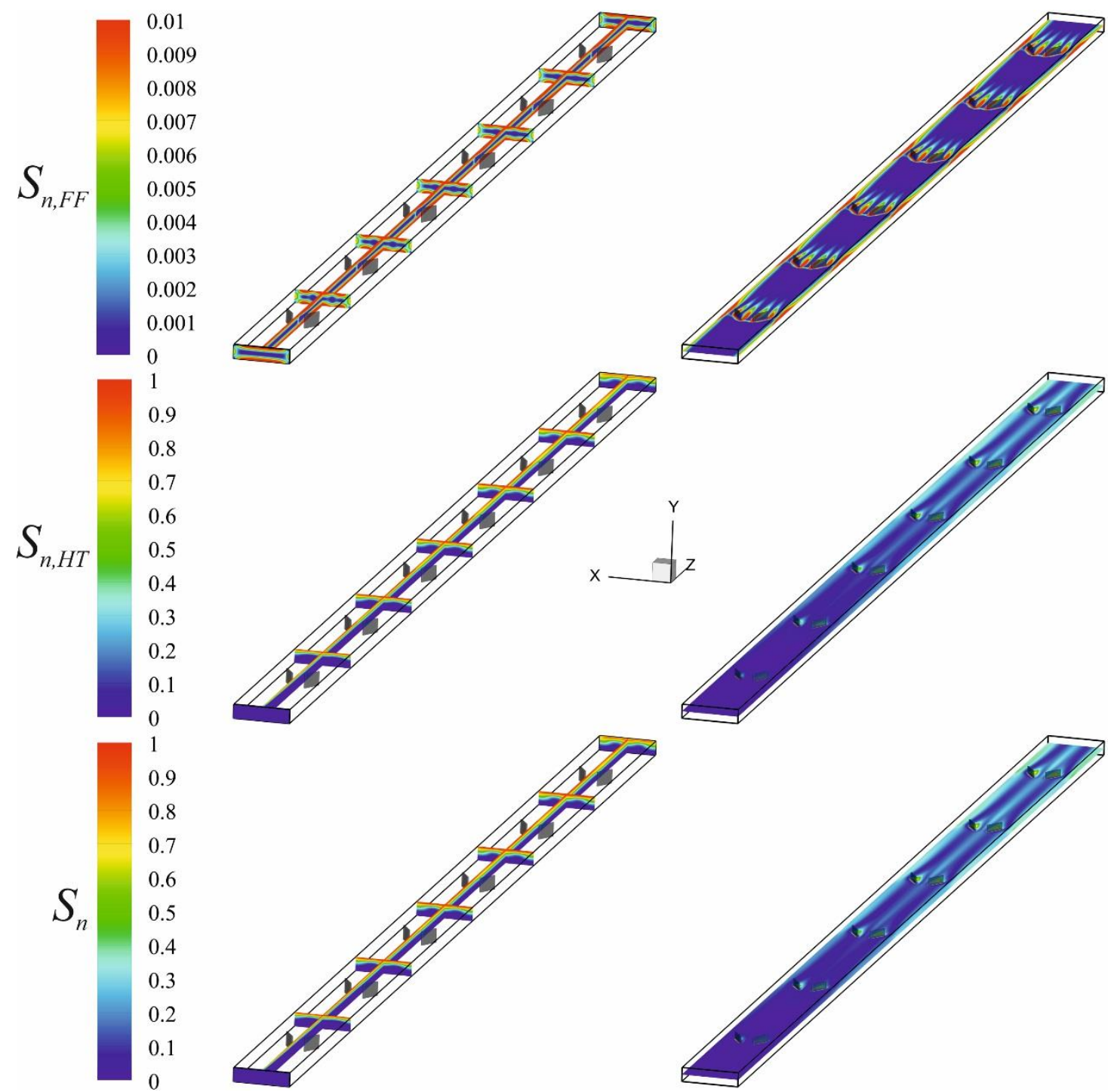

Fig. 9- Contours of dimensionless entropy generation due to fluid friction (top), heat transfer (middle) and both sources (bottom) for water-CuO flow with $\alpha=3 \%$ and $d_{p}=29.0 \mathrm{~nm}$ at $R e=150$. 